\title{
INFLUÊNCIA DOS CATALISADORES NA TOXICIDADE DE NANOTUBOS DE CARBONO
}

\section{Natália Zago Sentena ${ }^{1}$; Ingrid Rosales Costa ${ }^{2}$; Altevir Rossato Viana ${ }^{3}$;Cristiano Rodrigo Bohn Rhoden ${ }^{4}$;Sergio Roberto Mortari ${ }^{5}$}

\section{RESUMO}

O método de deposição quimica a vapor (CVD) é de grande interesse na produção de nanotubos de carbono, por ser um processo controlado e com baixa temperatura. A fonte da produção são os catalisadores em escala de 1-100 nm. Este trabalho tem como objetivo produzir catalisadores ( $\mathrm{Fe}, \mathrm{Co}, \mathrm{Ni}$, Co-Fe, Ni-Fe e Co-Ni-Fe) e avaliar a toxicidade destes materiais em NTCs, por meio de testes biológicos. A produção dos catalisadores segue adaptação de ZAMPIVA, et al. (2018), a partir da síntese de deposição química a vapor, dissolvendo percursores em uma solução aquosa $[(\mathrm{Fe}(\mathrm{NO} 3) 2.6 \mathrm{H} 2 \mathrm{O}, \quad \mathrm{Ni}(\mathrm{NO} 3) 2.6 \mathrm{H} 2 \mathrm{O}$ e $\mathrm{Co}(\mathrm{NO} 3) 2.6 \mathrm{H} 2 \mathrm{O}]$, com um agente complexante e combustível (Glicina). A purificação ocorre à $900^{\circ} \mathrm{C}$ por $30 \mathrm{~min}$. $\mathrm{O}$ CVD utiliza como gás inerte o Argônio, Hidrogênio como ativador e o etileno como fonte de carbono, em temperatura de síntese de $850^{\circ} \mathrm{C}$ por 40 minutos. A etapa de purificação utiliza tratamento térmico e químico. Foram realizadas caracterizações por Difração de Raios X (DRX) e ensaios in vitro de MTT. Foi observada a presença dos metais de transição nas amostras e também foi constatado um aumento no número de células e diminuição na viabilidade celular, respectivamente.

Palavras-chave: Nanomateriais, Óxidos Metálicos, Citotoxicidade.

Eixo Temático: Tecnologia, Inovação e Desenvolvimento Sustentável (TIDS)

\section{INTRODUÇÃO}

A produção de materiais que possuem em seu arranjo o carbono, como os nanotubos de carbono (NTC) é de amplo interesse, segundo Raphey e colaboradores (RAPHEY V.R. et al, 2019) isso se deve as suas propriedades estruturais, mecânicas e eletrônicas. Conforme Ali e colaboradores (ALI, et al, 2021) o método de deposição química a vapor é o mais apropriado para uma produção de NTC em escala industrial, devido a possibilidade de otimização nas condições do processo (temperatura, tempo de síntese, pressão).

\footnotetext{
${ }_{1}$ Natália Zago Sentena - Universidade Franciscana. natalia.zago@ufn.edu.br

${ }^{2}$ Ingrid Rosales Costa - Universidade Franciscana. ingrid.costa@ufn.edu.br.

${ }^{3}$ Altevir Rossato Viana - Universidade Franciscana. rossato.viana@hotmail.com.

${ }^{4}$ Cristiano Rodrigo Bohn Rhoden - Universidade Franciscana. cristianorbr@ufn.edu.br.

${ }^{5}$ Sergio Roberto Mortari - Universidade Franciscana. mortari@ufn.edu.br.
} 
O emprego deste método se faz necessário temperatura (800 - 1000 ${ }^{\circ} \mathrm{C}$ ), fonte de carbono (gás ou líquido) e catalisadores (geralmente metais de transição). Catalisadores de óxidos metálicos, produzidos a partir da síntese de combustão por solução, são bem aceitos, pois produzem partículas em escala nanométrica e grande área superficial (ZAMPIVA, et al, 2017).

A aplicação dos NTC como carreadores de fármacos ainda não é totalmente acessível, devido ao efeito de toxicidade, que pode levar a danos no DNA, induzir a formação de espécies reativas de oxigênio e a liberação de íons, prejudiciais aos componentes celulares (WISDOM K.S. et al, 2020). Tendo em vista a dificuldade do uso desse material em meio biológico, o presente trabalho avaliou a influência do tipo de catalisador na produção de NTC, quanto a toxicidade.

\section{METODOLOGIA}

\section{Produção dos catalisadores}

Conforme ZAMPIVA, et al. (2018), foram produzidos catalisadores ( $\mathrm{Fe}, \mathrm{Co}, \mathrm{Ni}$, $\mathrm{Co}-\mathrm{Fe}$, Ni-Fe e Co-Ni-Fe), pela síntese de combustão em solução. Foram empregados precursores em solução aquosa $\left(\mathrm{Fe}\left(\mathrm{NO}_{3}\right)_{2} \cdot 6 \mathrm{H}_{2} \mathrm{O}, \mathrm{Ni}\left(\mathrm{NO}_{3}\right)_{2} .6 \mathrm{H}_{2} \mathrm{O}\right.$ e $\left.\mathrm{Co}\left(\mathrm{NO}_{3}\right)_{2} \cdot 6 \mathrm{H}_{2} \mathrm{O}\right]$, um agente complexante e combustível (Glicina). Foi aplicado oxirredução e em seguida emparelhamos os nitratos com a Glicina de acordo com a tabela 1.

Tabela 1- Quantidade de reagente utilizado na produção de catalisadores pelo método de combustão em solução.

\begin{tabular}{lllllll}
\hline Reagente $(\mathrm{g})$ & \multicolumn{7}{l}{ Catalisador Formado } & \multicolumn{3}{l}{} \\
\hline \multirow{2}{*}{ Nitrato de Ferro } & 1 & 2 & 3 & 4 & 5 & 6 \\
\cline { 2 - 7 } Nitrato de Níquel & 24,24 & - & - & 16,00 & 16,00 & 16,00 \\
Nitrato de Cobalto & - & 8,70 & - & 1,50 & - & 5,00 \\
Glicina & 7,49 & - & 5,40 & - & 5,80 & 3,60 \\
\hline
\end{tabular}

Seguem as respectivas reações de síntese:

(1) $2 \mathrm{Fe}\left(\mathrm{NO}_{3}\right)_{3} .9 \mathrm{H}_{2} \mathrm{O}+6 \mathrm{C}_{2} \mathrm{H}_{5} \mathrm{NO}_{2}$

(2) $\mathrm{Ni}\left(\mathrm{NO}_{3}\right)_{2}+2 \mathrm{Fe}\left(\mathrm{NO}_{3}\right)_{3} .9 \mathrm{H}_{2} \mathrm{O}+\mathrm{C}_{2} \mathrm{H}_{5} \mathrm{NO}_{2}$

${ }^{1}$ Natália Zago Sentena - Universidade Franciscana. natalia.zago@ufn.edu.br

2 Ingrid Rosales Costa - Universidade Franciscana. ingrid.costa@ufn.edu.br.

3 Altevir Rossato Viana - Universidade Franciscana. rossato.viana@hotmail.com.

${ }^{4}$ Cristiano Rodrigo Bohn Rhoden - Universidade Franciscana. cristianorbr@ufn.edu.br.

${ }^{5}$ Sergio Roberto Mortari - Universidade Franciscana. mortari@ufn.edu.br. 
(3) $\mathrm{Co}\left(\mathrm{NO}_{3}\right)_{2}+2 \mathrm{Fe}\left(\mathrm{NO}_{3}\right)_{3} .9 \mathrm{H}_{2} \mathrm{O}+\mathrm{C}_{2} \mathrm{H}_{5} \mathrm{NO}_{2}$

(4) $\mathrm{Ni}\left(\mathrm{NO}_{3}\right)_{2}+2 \mathrm{Fe}\left(\mathrm{NO}_{3}\right)_{3} .9 \mathrm{H}_{2} \mathrm{O}+2 \mathrm{Fe}\left(\mathrm{NO}_{3}\right)_{3} .9 \mathrm{H}_{2} \mathrm{O} \mathrm{C} \mathrm{H}_{5} \mathrm{H}_{5} \mathrm{NO}_{2}$

(5) $\mathrm{Co}\left(\mathrm{NO}_{3}\right)_{2}+2 \mathrm{Fe}\left(\mathrm{NO}_{3}\right)_{3} .9 \mathrm{H}_{2} \mathrm{O}+2 \mathrm{Fe}\left(\mathrm{NO}_{3}\right)_{3} .9 \mathrm{H}_{2} \mathrm{O} \mathrm{C} \mathrm{C}_{2} \mathrm{H}_{5} \mathrm{NO}_{2}$

(6) $\mathrm{Ni}\left(\mathrm{NO}_{3}\right)_{2}+2 \mathrm{Fe}\left(\mathrm{NO}_{3}\right)_{3} .9 \mathrm{H}_{2} \mathrm{O}+\mathrm{Co}\left(\mathrm{NO}_{3}\right)_{2}+2 \mathrm{Fe}\left(\mathrm{NO}_{3}\right)_{3} .9 \mathrm{H}_{2} \mathrm{O}+2 \mathrm{Fe}\left(\mathrm{NO}_{3}\right)_{3} .9 \mathrm{H}_{2} \mathrm{O}$ $\mathrm{C}_{2} \mathrm{H}_{5} \mathrm{NO}_{2}$

No procedimento adotado, os nitratos metálicos foram solubilizados e agitados separadamente, com o mínimo de água e então misturados e agitados por 5 minutos, conforme figura 1 (a). A glicina foi solubilizada e agitada com um leve aquecimento. Em seguida, a Glicina foi misturada com os nitratos mantendo a agitação por mais 10 minutos.
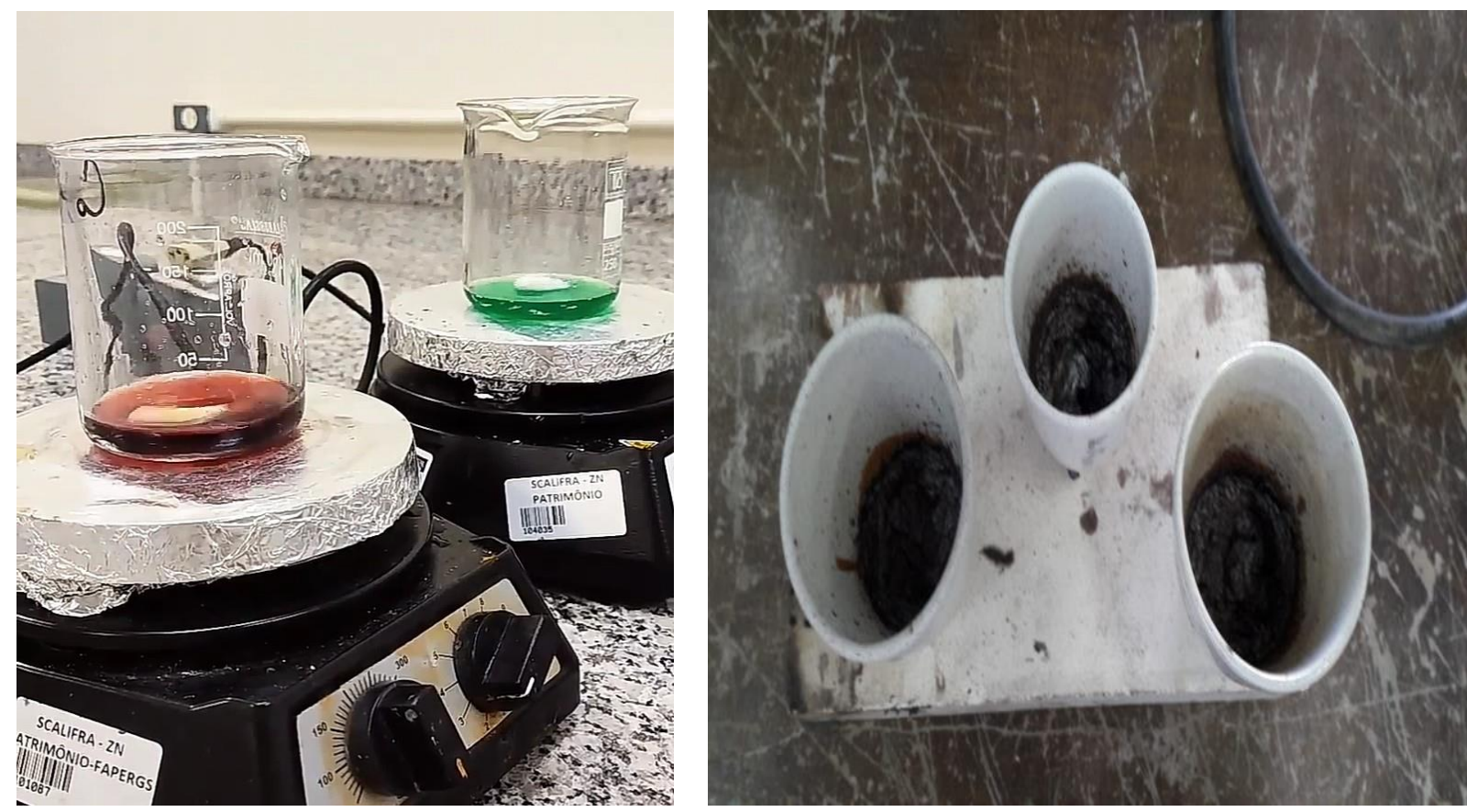

Figura 1: (a) preparo dos reagentes; (b) Catalisador formado após a etapa de combustão (400 $\stackrel{\circ}{ } \mathrm{C}$, por 30 minutos).

As soluções foram colocadas em um equipamento para aquecimento, préaquecido a $200^{\circ} \mathrm{C}$ e a temperatura foi elevada para $400^{\circ} \mathrm{C}$ (figura 1 (b)), até que houvesse a combustão completa (cerca de 30min). Os materiais foram resfriados até atingirem a temperatura ambiente e, em seguida macerados e sinterizados em temperatura de $900^{\circ} \mathrm{C}$, também em mufla por 30 minutos.

1 Natália Zago Sentena - Universidade Franciscana. natalia.zago@ufn.edu.br

2 Ingrid Rosales Costa - Universidade Franciscana. ingrid.costa@ufn.edu.br.

${ }^{3}$ Altevir Rossato Viana - Universidade Franciscana. rossato.viana@hotmail.com.

${ }^{4}$ Cristiano Rodrigo Bohn Rhoden - Universidade Franciscana. cristianorbr@ufn.edu.br.

${ }^{5}$ Sergio Roberto Mortari - Universidade Franciscana. mortari@ufn.edu.br. 


\section{Produção dos nanotubos de carbono}

Para a obtenção de NTC, por CVD, 100 mg de cada catalisador produzido, foram pesados diretamente em barquinhas de porcelana e colocadas no forno (Jung, modelo LT3.9013, com temperatura de operação máxima de $1300{ }^{\circ} \mathrm{C}$ ), figura 2.0 reator de síntese foi um tubo de quartzo com 1,20 m de comprimento e diâmetro interno de $32 \mathrm{~mm}$. Os fluxos dos gases foram controlados por um fluxômetro múltiplo (Hidrogênio/Argônio/Etileno) da Aalborg-USA, modelo P31S1VA1. O Argônio foi liberado constantemente no meio até que a temperatura atingiu $600^{\circ} \mathrm{C}$, então 0 hidrogênio foi liberado como gás ativador dos catalisadores. $\mathrm{E}$ atingindo $850^{\circ} \mathrm{C} \mathrm{O}$ etileno, como gás fonte de carbono é liberado por 40min.

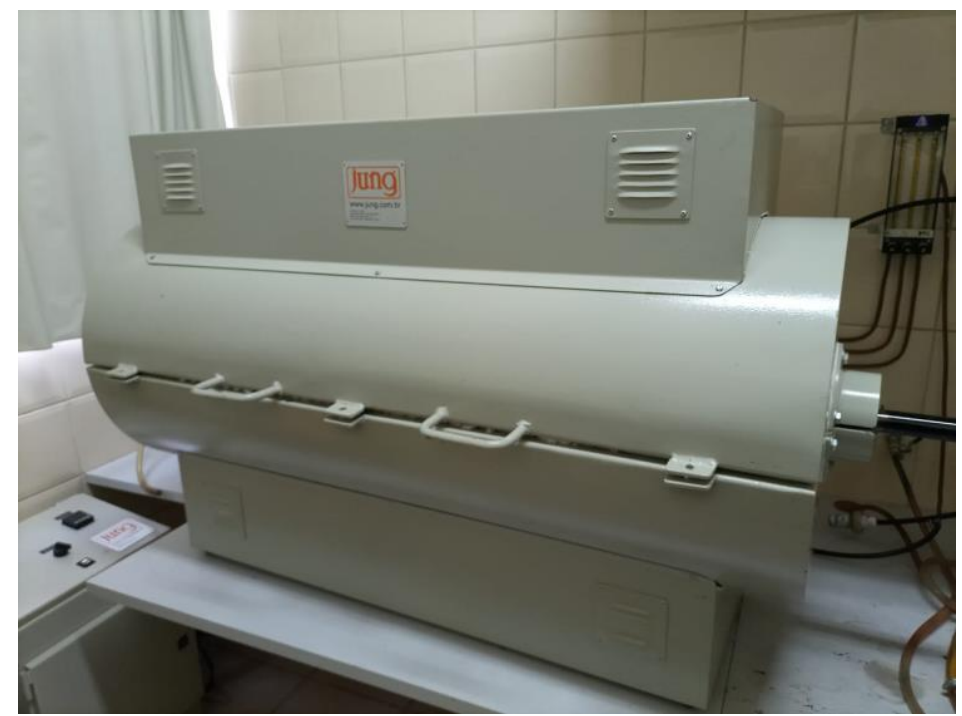

Figura 2: forno da produção dos NTCs.

Para a purificação dos nanotubos produzidos (eliminação de carbono amorfo e resíduos de catalisador) utilizou-se tratamento térmico (400 ${ }^{\circ} \mathrm{C}$ por 30 minutos) e químico em meio clorídrico - $\mathrm{HCl} 4$ mol.L-1 a $60^{\circ} \mathrm{C}$ por 120 minutos. Após filtração e sucessivas lavagens com água até atingir pH neutro (figura 3) para então, secar em estufa e armazenar.

${ }_{1}$ Natália Zago Sentena - Universidade Franciscana. natalia.zago@ufn.edu.br

2 Ingrid Rosales Costa - Universidade Franciscana. ingrid.costa@ufn.edu.br.

${ }^{3}$ Altevir Rossato Viana - Universidade Franciscana. rossato.viana@hotmail.com.

${ }^{4}$ Cristiano Rodrigo Bohn Rhoden - Universidade Franciscana. cristianorbr@ufn.edu.br.

${ }^{5}$ Sergio Roberto Mortari - Universidade Franciscana. mortari@ufn.edu.br. 


\section{QUFN}

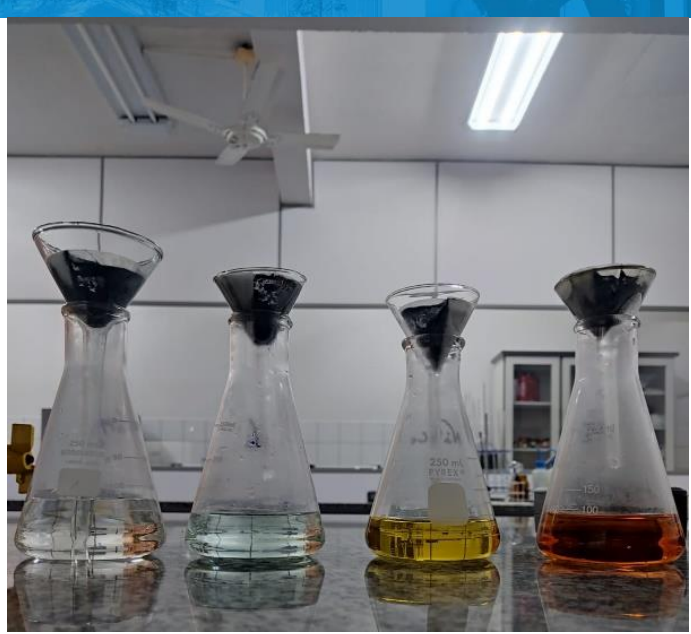

Figura 3 - Purificação química dos NTC produzidos.

\section{Ensaio In vitro}

Para o ensaio de MTT a enzima succinate desidrogenase presente em células viáveis que metabolizam o reagente MTT é adicionado nos poços $20 \mu \mathrm{L}(5 \mathrm{mg} / \mathrm{mL}$ de PBS) onde é convertido em MTT Formazan, solubilizado e sua concentração determinada por densidade óptica, após 4 horas de incubação. O sobrenadante é retirado e então adicionado $200 \mu \mathrm{L}$ de dimetilsulfóxido para solubilizar os cristas de cor púrpura. O ensaio é feito em microplacas de Elisa de 96 poços.

\section{RESULTADOS E DISCUSSÕES}

\section{Difração de Raios X (DRX) ensaio In vitro}

Foram realizados testes de DRX e ensaio in vitro nas amostras de catalisadores e NTCs. A analise foi feita em grupos, comparando os resultados do catalisador com o seu produto NTC. Nos testes de DRX todos apresentaram seus picos caracteristicos e no ensaio in vitro ocorreu variação na citotoxicidade conforme cada tipo de amostras. O padrão que se sobressaio foi do NiFe, não apresentando significativa variação nos testes de viabilidade celular.

${ }^{1}$ Natália Zago Sentena - Universidade Franciscana.natalia.zago@ufn.edu.br

2 Ingrid Rosales Costa - Universidade Franciscana. ingrid.costa@ufn.edu.br.

3 Altevir Rossato Viana - Universidade Franciscana. rossato.viana@hotmail.com.

${ }^{4}$ Cristiano Rodrigo Bohn Rhoden - Universidade Franciscana. cristianorbr@ufn.edu.br.

${ }^{5}$ Sergio Roberto Mortari - Universidade Franciscana. mortari@ufn.edu.br. 


\section{QUFN}

a)

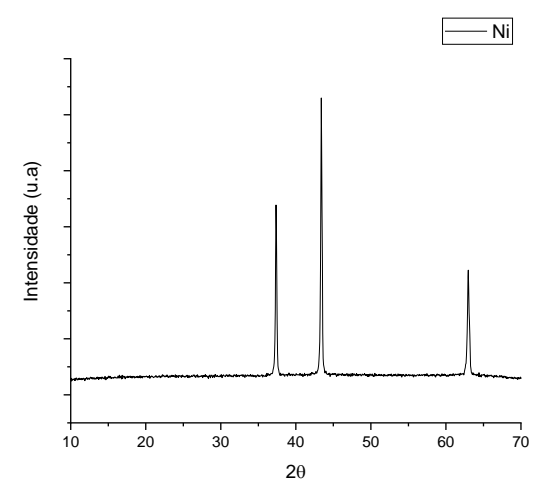

HaCat 24h

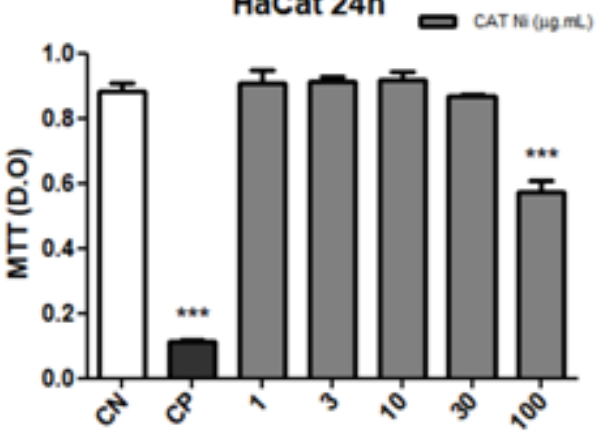

b)
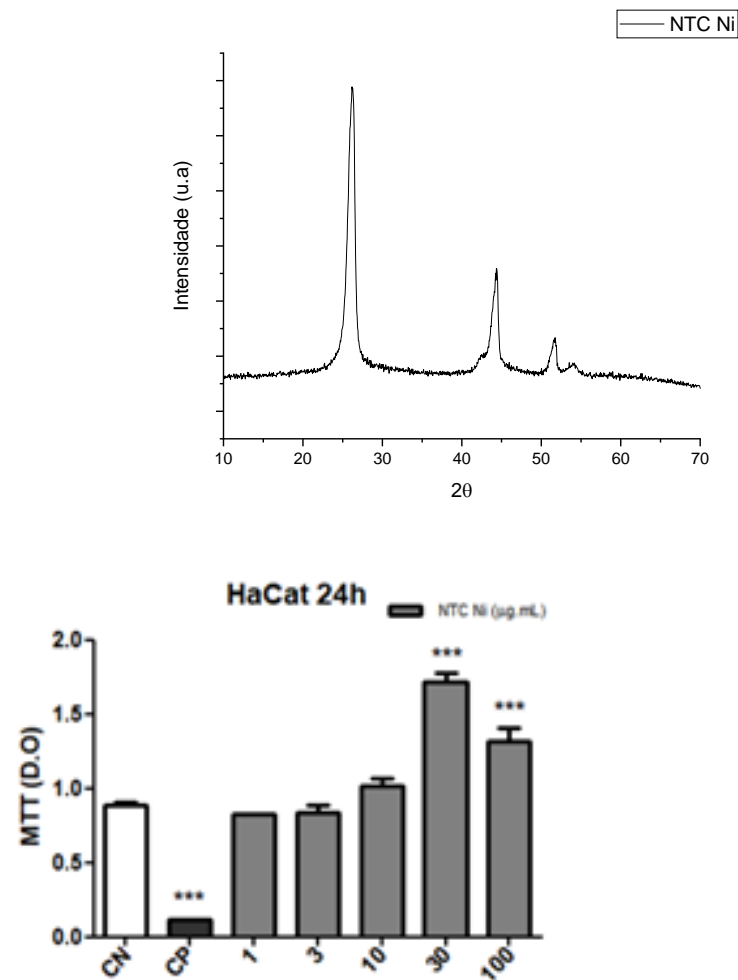

Figura 4- (a) Difratograma e teste in vitro catalisador $\mathrm{Ni}$ (b) Difratograma e teste in vitro NTC Ni

Constatamos nos difratogramas os picos caracteristicos do $\mathrm{Ni} 44^{\circ}$, além disso, podemos observar nos testes MTT que o catalisador de Ni sofreu diminuição na viabilidade celular em 100ul e os NTC em 30ul ocorreu inflamação celular.

${ }^{1}$ Natália Zago Sentena - Universidade Franciscana.natalia.zago@ufn.edu.br

2 Ingrid Rosales Costa - Universidade Franciscana. ingrid.costa@ufn.edu.br.

3 Altevir Rossato Viana - Universidade Franciscana. rossato.viana@hotmail.com.

${ }^{4}$ Cristiano Rodrigo Bohn Rhoden - Universidade Franciscana. cristianorbr@ufn.edu.br.

${ }^{5}$ Sergio Roberto Mortari - Universidade Franciscana. mortari@ufn.edu.br. 
c)
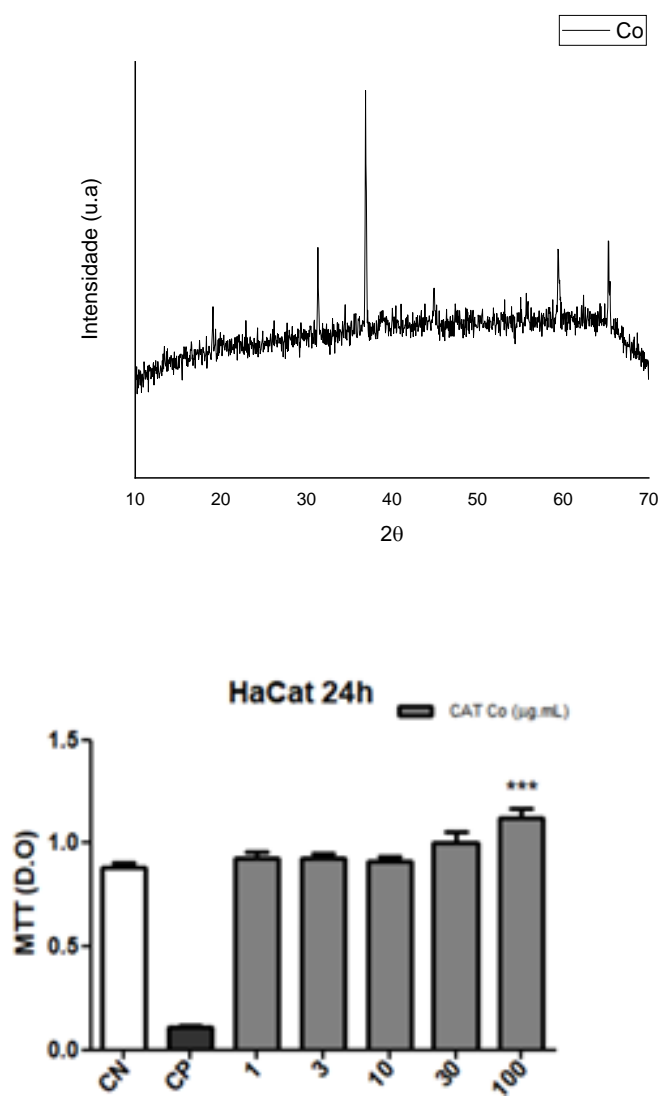

d)
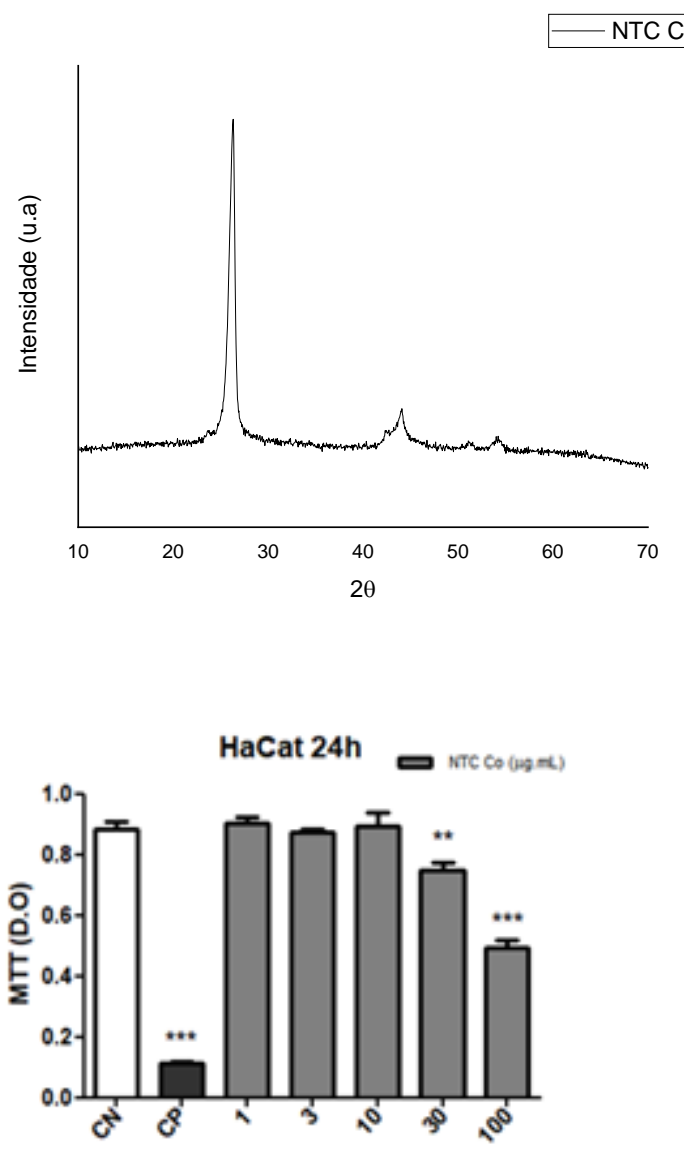

Figura 5 - (c) Difratograma e teste in vitro catalisador Co (d) Difratograma e teste in vitro NTC Co

As caracterizações de DRX apresentaram os picos caracteristicos do Co $38^{\circ}$, no MTT o catalisador sofreu inflamação em 100ul e o NTC sofreu diminuição na viabilidade celular a partir de 30 ul.

${ }^{1}$ Natália Zago Sentena - Universidade Franciscana.natalia.zago@ufn.edu.br

2 Ingrid Rosales Costa - Universidade Franciscana. ingrid.costa@ufn.edu.br.

3 Altevir Rossato Viana - Universidade Franciscana. rossato.viana@hotmail.com.

${ }^{4}$ Cristiano Rodrigo Bohn Rhoden - Universidade Franciscana. cristianorbr@ufn.edu.br.

${ }^{5}$ Sergio Roberto Mortari - Universidade Franciscana. mortari@ufn.edu.br. 


\section{QUFN}

e)

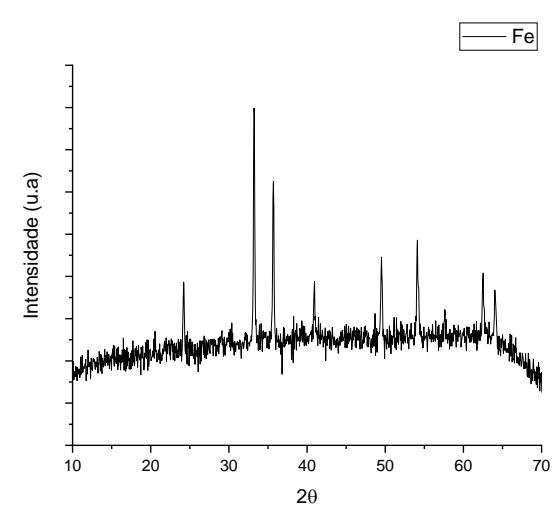

HaCat 24h

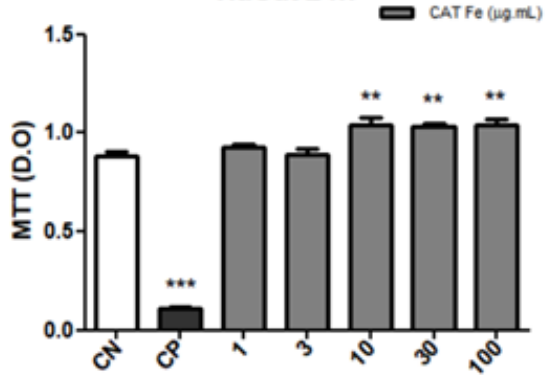

f)
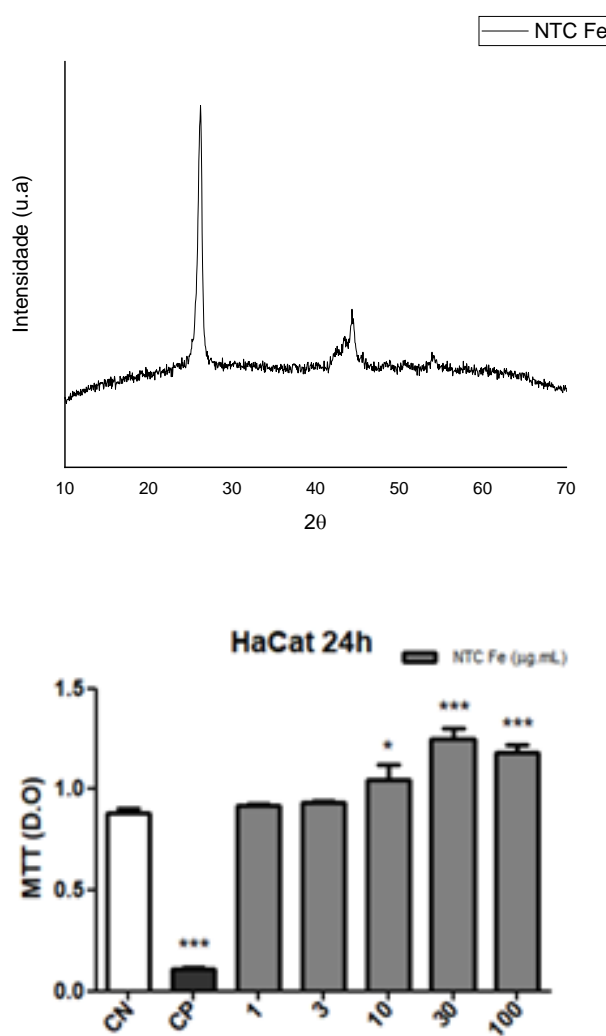

Figura 6- (e) Difratograma e teste in vitro catalisador Fe (f) Difratograma e teste in vitro NTC Fe

Os difratogramas apresentaram os picos caracteristicos do $\mathrm{Fe}$ de $34^{\circ}$. Os testes MTT tanto para o catalisador como para o NTC Fe ocorreu inflamação a partir de 10ul.

g)



h)

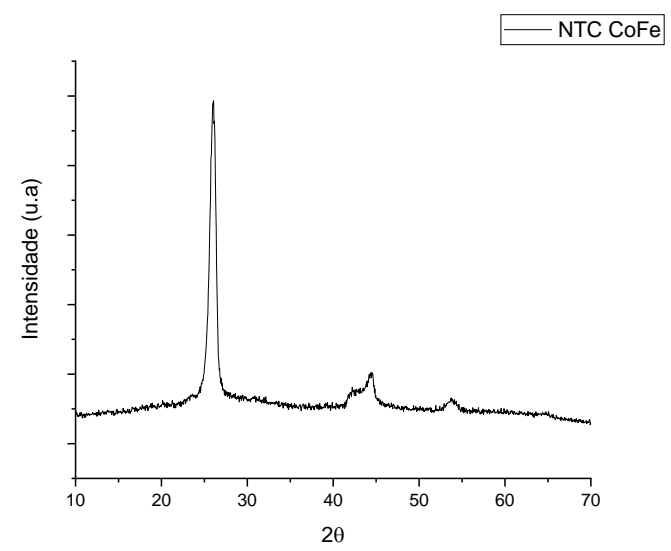

${ }^{1}$ Natália Zago Sentena - Universidade Franciscana.natalia.zago@ufn.edu.br

2 Ingrid Rosales Costa - Universidade Franciscana. ingrid.costa@ufn.edu.br.

3 Altevir Rossato Viana - Universidade Franciscana. rossato.viana@hotmail.com.

${ }^{4}$ Cristiano Rodrigo Bohn Rhoden - Universidade Franciscana. cristianorbr@ufn.edu.br.

${ }^{5}$ Sergio Roberto Mortari - Universidade Franciscana. mortari@ufn.edu.br. 

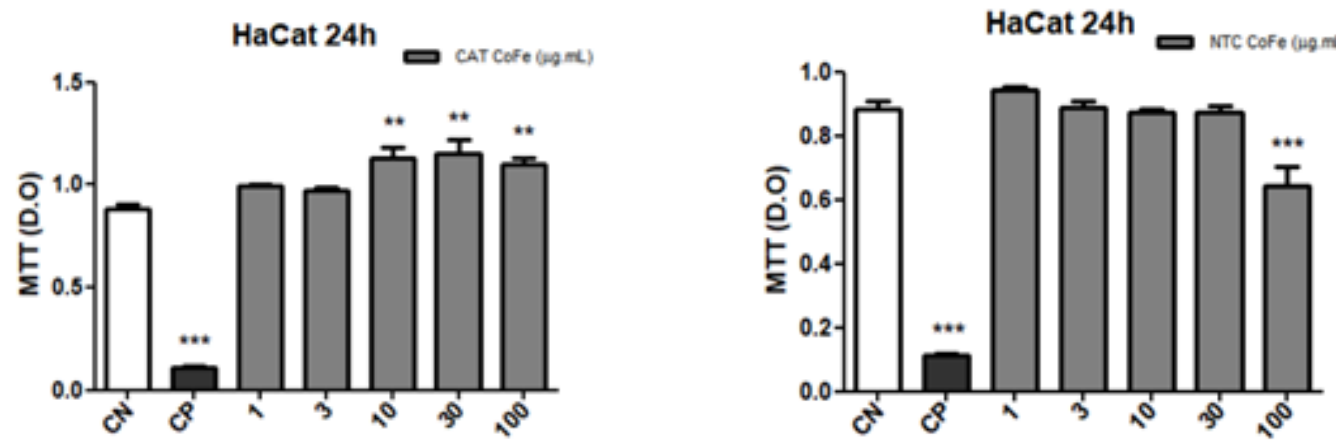

Figura 7 - (g) Difratograma e teste in vitro catalisador CoFe (h) Difratograma e teste in vitro NTC $\mathrm{CoFe}$

$\mathrm{Na}$ caracterização por DRX apresentou os picos de $38^{\circ} \mathrm{Co}$ e $34^{\circ} \mathrm{Fe}$. Os testes MTT, para o catalisador demostrou inflamação celular a partir da 10ul e para o NTC diminuição na viabilidade celular 100ul.

i)

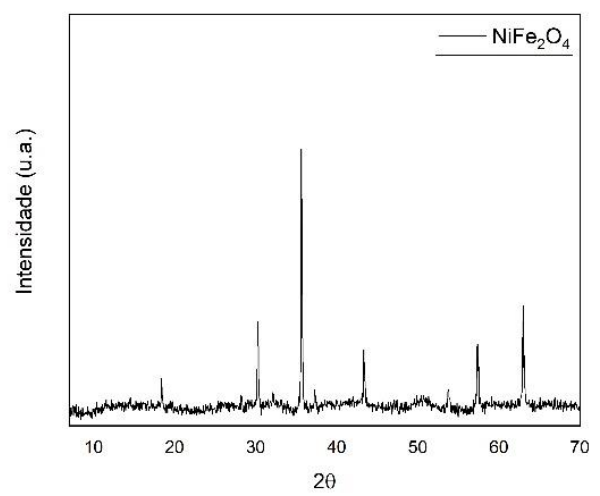

HaCat 24h $\square$ carmage (ugm)

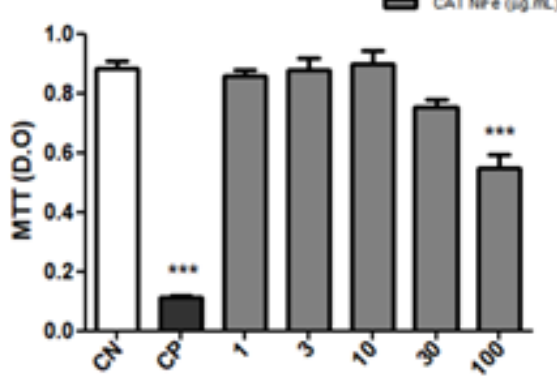

J)

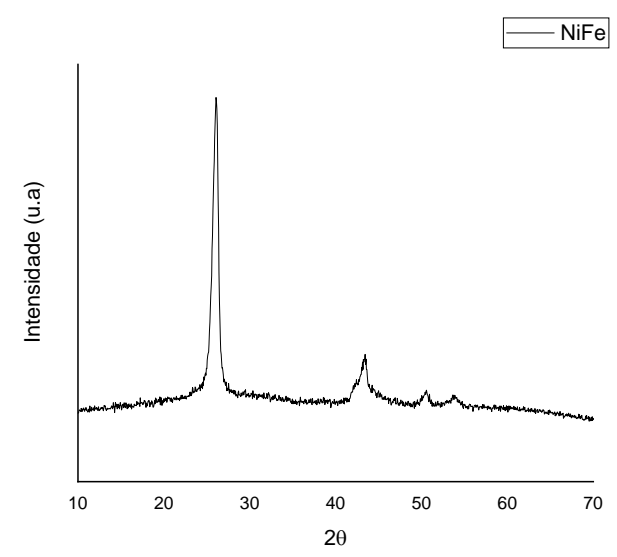

HaCat 24h

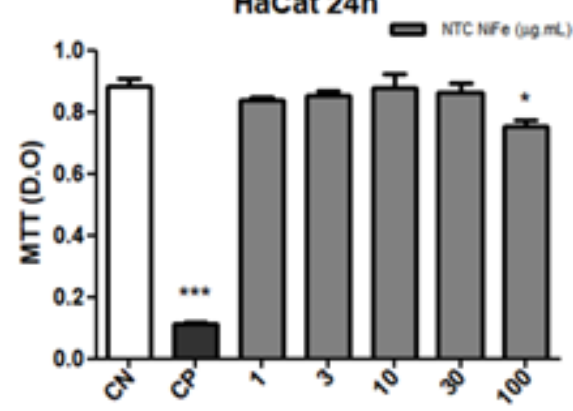

Figura 8- (i) Difratograma e teste in vitro catalisador NiFe (j) Difratograma e teste in vitro NTC NiFe

Os digratogramas apresentaram os picos caracteristicos dos materiais, para Ni $44^{\circ} \mathrm{e}$ para $\mathrm{Fe} 34^{\circ}$. Nos testes de viabilidade celular MTT, o catalisador apresentou diminuição na viabilidade celular a partir de 30ul, já o NTC apresentou diminuição na viabilidade celular em 100ul.

${ }^{1}$ Natália Zago Sentena - Universidade Franciscana.natalia.zago@ufn.edu.br

2 Ingrid Rosales Costa - Universidade Franciscana. ingrid.costa@ufn.edu.br.

3 Altevir Rossato Viana - Universidade Franciscana. rossato.viana@hotmail.com.

${ }^{4}$ Cristiano Rodrigo Bohn Rhoden - Universidade Franciscana. cristianorbr@ufn.edu.br.

${ }^{5}$ Sergio Roberto Mortari - Universidade Franciscana. mortari@ufn.edu.br. 
k)

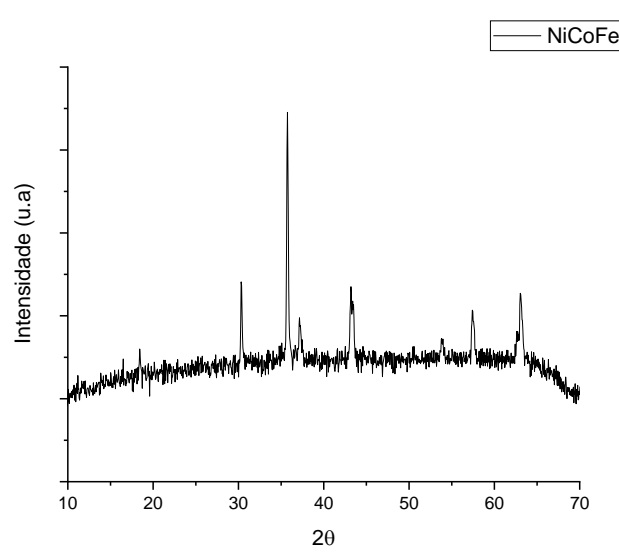

HaCat 24h

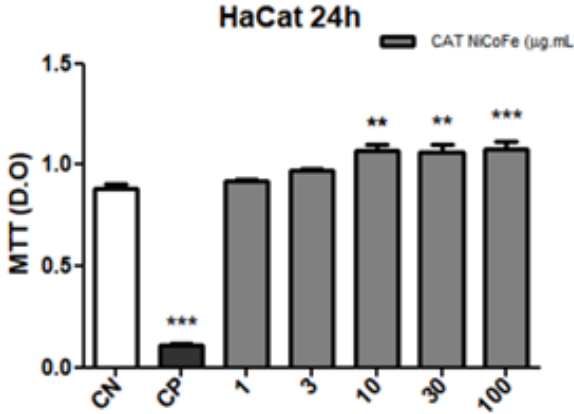

l)
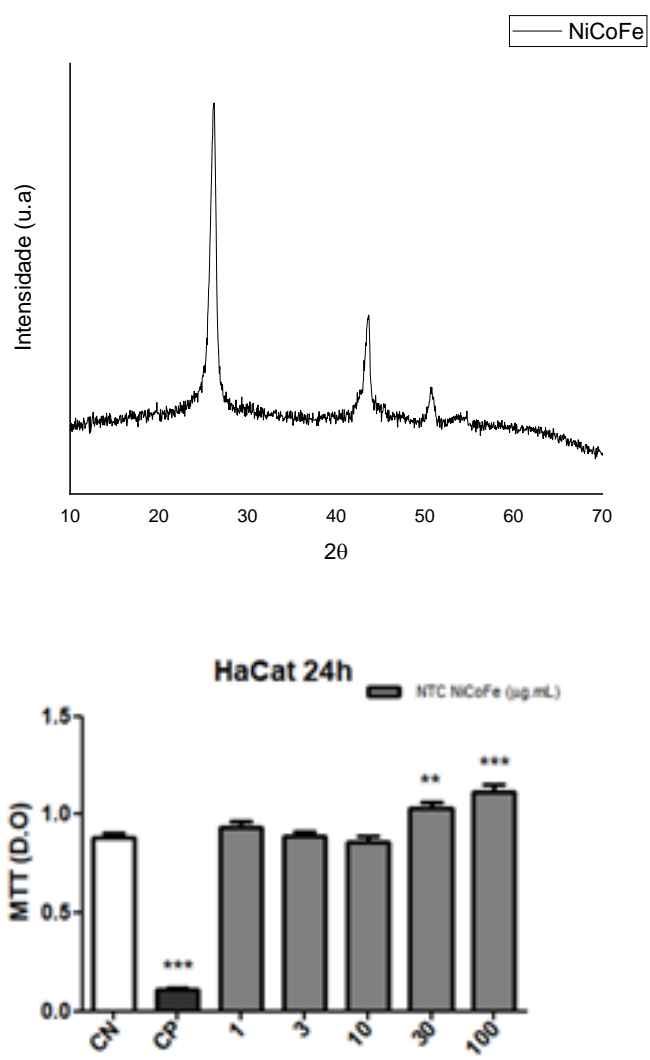

Figura 9-(k) Difratograma e teste in vitro catalisador NiCoFe (I) Difratograma e teste in vitro NTC $\mathrm{NiCoFe}$

A caracterização por DRX apresentou os picos característicos do $\mathrm{Ni} 44^{\circ}$, Co $38^{\circ} \mathrm{e}$ Fe $34^{\circ}$. Nos testes de MTT no catalisador ocorreu inflamação a partir de $10 \mathrm{ul}$ e o NTC também apresentou inflamação porem a partir de 30ul.

\section{CONCLUSÃO}

Os resultados dos testes de DRX apresentaram picos característicos, confirmando a eficiência na produção dos catalisadores e NTC. Os testes de viabilidade MTT apresentaram diminuição na viabilidade celular e inflamação celular, com destaque nas amostras NiFe, que não apresentaram grande variação nos testes MTT.

\section{AGRADECIMENTOS CNPq e UFN}

${ }^{1}$ Natália Zago Sentena - Universidade Franciscana.natalia.zago@ufn.edu.br

2 Ingrid Rosales Costa - Universidade Franciscana. ingrid.costa@ufn.edu.br.

3 Altevir Rossato Viana - Universidade Franciscana. rossato.viana@hotmail.com.

${ }^{4}$ Cristiano Rodrigo Bohn Rhoden - Universidade Franciscana. cristianorbr@ufn.edu.br.

${ }^{5}$ Sergio Roberto Mortari - Universidade Franciscana. mortari@ufn.edu.br. 


\section{QUFN}

\section{REFERÊNCIAS}

ALI, I. et al. A new approach to the economic synthesis of multi-walled carbon nanotubes using a Ni/MgO catalyst. Materials Chemistry and Physics, p. 1-8, 2021.

RAPHEY, V. R. et al. Advanced biomedical applications of carbon nanotube. Materials Science \& Engineering C, p. 616-630, 2019.

WISDOM K. S. et al. Chitosan grafting onto single-walled carbon nanotubes increased their stability and reduced the toxicity in vivo (catfish) model. International Journal of Biological Macromolecules, p. 697-707, 2020.

ZAMPIVA, R., Y., S. et al. 3D CNT macrostructure synthesis catalyzed by MgFe2O4 nanoparticles: A study of surface area and spinel inversion influence. Applied Surface Science, v. 422, p. 321-330, 2017.

ZAMPIVA, R., Y., S. et al. Influence of the Fuel Composition and the Fuel/Oxidizer Ratio on the Combustion Solution Synthesis of MgFe2O4 Catalyst Nanoparticles. FME Transactions v. 46, p. 157-164, 2018. 